\title{
The Protective Role of Date Palm (Phoenix Dactylifera Seeds) against Aflatoxicosis in Broiler Chickens Regarding Carcass Characterstics, Hepatic and Renal Biochemical Function Tests and Histopathology
}

\author{
Ward Masoud Abdel-Sattar ${ }^{1}$, Kadry Mohamed Sadek ${ }^{2}$, Ahmed Ragab Elbestawy ${ }^{3}$ and Disouky Mohamed Mourad ${ }^{1^{*}}$ \\ ${ }^{\prime}$ Department of Animal and Poultry Health, Division of Animal and Poultry Production, Desert Research Center, Cairo, 2633759, Egypt. \\ ${ }^{2}$ Department of Biochemistry, Faculty of Veterinary Medicine, Damanhour University, Damanhour, 22511, Egypt. \\ ${ }^{3}$ Department of Poultry and Fish Diseases, Faculty of Veterinary Medicine, Damanhour University, Damanhour, 22511, Egypt. \\ *Corresponding author`s Email: dismou235@gmail.com; ORCID: 0000-0001-7777-9305
}

\begin{abstract}
Harmful effects caused by aflatoxin (AF) directed researchers towards to find out new strategies for its control and detoxification increasing the safety of poultry feed. The aim of the present work was to study the protective role of date pits (Phoenix dactylifera) seeds against aflatoxicosis regarding carcass traits, biochemical function tests and histopathology of both liver and kidney in broiler chickens. 210 one-day old Arbor Acres broiler chicks were allotted into 7 equal groups as the first control (G1) supplemented by the basal diet, G2 had the basal diet with date pits supplementation $2 \%$, G3 fed on the basal diet with date pits 4\%, G4 was fed a basal diet containing $100 \mu \mathrm{g}$ aflatoxin/kg (100 ppb). G5 fed on a basal diet containing Hydrated Sodium Calcium Aluminum Silicates (HSCAS) $0.3 \%$ plus aflatoxin, (G6) fed a basal diet containing date pits $2 \%$ plus aflatoxin and finally G7 fed a basal diet containing date pits $4 \%$ plus aflatoxin. The aflatoxin supplemented to the broiler ration from first day to the end of experiment at 35 days. Aflatoxins supplementation significantly increased relative liver and small intestine weight, affect liver and kidney biochemical function tests and induced histopathological changes as fatty degeneration of hepatocytes, and interstitial nephritis with mononuclear cell infiltrations in both liver and kidney, respectively. However, addition of date pits $(2 \%$ and $4 \%)$ and HSCAS $(0.3 \%)$ to broiler's diet partially ameliorated these harmful effects of aflatoxins, indicating their protective effect against aflatoxicosis and this protection is dose-related. Addition of date palm seed (2\% and $4 \%$ ) gave a better results regarding carcass traits, biochemical parameters and histopathological examination of liver and kidney, finally concluding that date palm seed powder could be used as an effective feed additive to control aflatoxicosis in poultry with avoiding harmful effect of chemical mycotoxin binders (HSCAS)
\end{abstract}

Key words: Aflatoxins, Broilers, Biochemical traits, Carcass characteristics, Date palm, Histopathological changes.

\section{INTRODUCTION}

Aflatoxins are belonging to a heterologous group of fungal secondary metabolites called mycotoxins that adversely affecting human and animal health. Aflatoxins have been most commonly produced by strains of Aspergillus flavus, A. parasiticus, and A. nominus. Also, many other Aspergilli including Emericella teleomorphs have aflatoxigenic capabilities. They are named according to their blue or green fluorescence under UV light, there are four primary aflatoxins: AFB1, AFB2, AFG1, and AFG2. AFB1 is the most hepatotoxic, mutagenic, and prevalent worldwide (Rawal et al., 2010).

In poultry, consumption of AFB1 cause huge economic losses by retarding bird growth, increasing feed efficiency, damage to internal organs as liver and kidney, increased leg problems, increased the incidence of secondary infections leading to increased mortalities and carcass condemnation (Bintvihok and Kositcharoenkul, 2006). Additionally, it causes immuno-suppression and changes in relative organs weight (Kubena et al., 1993). Aflatoxins cause a wide range of metabolic changes in poultry associated with reduced digestive enzyme activities, and immunosuppression (Edds and Bortell, 1983).

Inorganic absorbents, such as hydrated sodium calcium aluminosilicates (HSCAS), sodium bentonite, zeolites or super-activated charcoal, can bind the aflatoxin before its absorption in the gastrointestinal tract and are shown to effectively reduce aflatoxicosis (Edrington et al., 1996), and addition of dietary chemicals such as ammonium hydroxide, calcium hydroxide, hydrogen peroxide, sodium hydroxide, and sodium hypochlorite is a stratgey to detoxify ingested aflatoxin (Jalili et al., 2011), all of which can reduce AFB1 concentrations through hydrolysis and can produce a degraded form with reduced or no toxicity. Although, most of these chemicals are often expensive and can lead to decrease the nutrient value of feed components. 
Phoenix dactylifera (date palm seeds) is a useful traditional medicinal plant which belongs to the family Arecaceae (Sirisena et al., 2015). The genus Phoenix contained 14 species including $P$. dactylifera that were cultivated in the Middle East for at least 6000 years (Copley et al., 2001). The global production of date fruits could be approximately 7 million tons in 2010. Egypt, Iran and Saudi Arabia were being the main producing countries, with a production of 1.13 million ton, 1 million ton and 983,000 ton, respectively (FAO, 2010).

Date palm seeds (DPS) are a by-product of many date manufacturing processes, such as for date syrup and date confectionery. This by-product is having a high-fiber source. DPS can be mostly discarded or used as a form of roughage for domestic farm animals (Golshan Tafti et al., 2017). Addition of DPS powder to animal, poultry and fish feed has been demonstrated to enhance growth, improve feed efficiency and meat palatability (Al-Farsi and Lee, 2011). Glucomannan commonly has the ability to biologically inactivates multiple mycotoxins and glucomannan type A is the main component of the cell walls of palm kernels, which in this case acts as a food reserve and disappears during germination (Navid, 2007).

The nutritional value of DPS may introduce a source of healthy feed for animals and poultry nutrition and an alternative agent to reduce the cost of feed additive chemical mycotoxin binders used for aflatoxin decontamination in poultry feed and many studies have been carried out on date seeds in Egypt focusing mainly on their chemical composition but, lacking the effects of these seeds on aflatoxicosis in poultry farms. Therefore, the use of $P$. dactylifera to ameliorate the harmful effects of aflatoxicosis in broiler have been investigated in the current experiment through evaluation of broiler carcass traits, hepatic and renal biochemical function tests and histopathology in comparison to HSCAS $(0.3 \%)$ supplementation in feed.

\section{MATERIALS AND METHODS}

\section{Production of aflatoxin}

Aflatoxin production was done using Aspergillus parasiticus NRRL - 2999 pure culture (National Research Centre, Cairo, Egypt) via fermentation of rice (Shotwell et al., 1966). The rice powder was incorporated into the basal diet at an estimated $100 \mu \mathrm{g} / \mathrm{kg}$ (100 ppb) entire the experimental period (35 days).

\section{Feed additives (Mycotoxin binders)}

\section{Phoenix dactylifera seeds collection and preparation}

It was purchased from a local date palm factory; washed, air-dried and finally grinded into a coarse powder and used as $2 \%$ and $4 \%$ mixed with basal diet according to each treatment.

\section{Hydrated sodium and calcium aluminum Silicates (HSCAS)}

A commercial mycotoxin adsorbent (Toxi-Mold Plus ${ }^{\circledR}$ ) obtained from Egyco-Vet Company was mixed with basal diet through a dose of $3 \mathrm{~kg}$ per ton for their bird groups.

\section{Experimental design}

Two hundred and ten 1-day-old unsexed Arbor Acres broiler chicks were purchased from a local commercial hatchery (NASCO Egypt, Alexandria) and randomly allocated into 7 equal groups at the first day of age. Each one group was subdivided into three replicates (10 birds per replicate) and floor reared. Feed (starter feed from $1^{\text {st }}$ to $21^{\text {st }}$ days and grower feed from $22^{\text {nd }}$ to $35^{\text {th }}$ days) and water were supplied ad-libitum for 35 days of age and optimum managemental factors were applied regarding ventilation, temperature, lighting and litter management all over the experimental period.

The diet composition was formulated according to the recommendation of National Research Council Nutrient Requirements for Arbor Acres broiler chickens NRC (1994) without any feed additives rather than the compounds under study. A basal diet and 6 treatment diets were used in 7 groups as follows: the first group (G1) fed on a commercial broiler diets without supplement (control); G2 (DPS2\%) fed on the basal diet with date pits $2 \%$ supplementation, G3 (DPS4\%) fed on the basal diet with date palm 4\%, G4 (AF) had aflatoxin as 100 $\mu \mathrm{g} / \mathrm{kg}$, G5 (AF+HSCAS) fed a basal diet containing HSCAS $0.3 \%$ plus aflatoxin, G6 (AF+DPS2\%) had a basal diet containing date palm $2 \%$ plus aflatoxin, and finally G7 (AF+DPS4\%) had a basal diet containing date pits 4\% plus aflatoxin. Also, the basal diets were tested for possible residual AF before start feeding and mixing the toxic dose and there were no detectable levels present. A recorded daily observation for health problems and mortality were carried out all over 35 days of age.

\section{Ethical approval}

The present study is affirmed by the Ethics of Animal Experiments Committee, Damanhour University, Egypt.

\section{Carcass characteristics}

At the end of experiment, five chickens in each group were randomly chosen, weighed, and humanely euthanized by cervical dislocation. After slaughtering of birds; abdominal fat, liver, pancreas, gizzard, heart, proventriculus, small 
intestine and lymphoid organs (bursa of Fabricius, thymus and spleen) were removed, weighed immediately and then; calculated as a percentage of carcass weight and finally, dressing percentage was calculated.

\section{Biochemical function tests}

The Blood samples were collected from wing vein by venous puncture at $2^{\text {nd }}, 3^{\text {rd }}, 4^{\text {th }}$ and $5^{\text {th }}$ weeks by using a sterile syringe in clean dry non-coated tubes. Each blood sample was left to coagulate at room temperature and centrifuged at $3000 \mathrm{rpm}$ for 5 minutes and clear serum was collected individually in Eppendorf tubes. The collected sera were subjected to determination of ALT, AST, GGT, ALP, bilirubin, uric acid, creatinine, total cholesterol, triacylglycerol, LDL and HDL following the instructions enclosed in the manufactured kits produced by Biodiagnostic Company, Egypt.

\section{Histopathological examination of the liver and kidney}

At the end of experiment on day 35, three chickens from each group were euthanized, liver and kidney samples were obtained and submitted for histopathology to evaluate lesions and abnormalities. Fixation of samples was applied in $10 \%$ buffered formalin solution for one week. Blocks and staining were carried out according to (Drury and Wallington, 1980).

\section{Statistical analysis}

Data was analyzed by one-way analysis of variance (ANOVA), with Duncan's multiple range tests for significant between means $(P \leq 0.05)$ by SPSS.20 ${ }^{\circledR}$ (IBM Cooperation, Armonk, NY, USA).

\section{RESULTS}

\section{Carcass traits in chicks aged 35 days of old}

The data illustrated in table 1 represented that dressing percentage of broilers are non-significantly $(\mathrm{P} \geq 0.05)$ affected in all treated groups in relation to negative control (G1), despite chicks received DPS2\% (G2) have the highest dressing \% and chicks received AF alone (G4) have the lowest dressing \% when compared to the control (G1) (61.2 \pm 0.01 and $50.3 \pm 0.01$ versus $58.3 \pm 0.01$, respectively).

There were non-significant differences $(\mathrm{P} \geq 0.05)$ among the experimental groups in most measured percentages of relative weights of organs, except only small intestine and liver. The percentage of relative weight of small intestine of broilers was decreased significantly $(\mathrm{P} \leq 0.05)$ in $\mathrm{G} 4(\mathrm{AF})$ and non-significantly $(\mathrm{P} \geq 0.05)$ in G5 and G6 when compared to control, while it was increased significantly $(\mathrm{P} \leq 0.05)$ in $\mathrm{G} 7$ and non-significantly $(\mathrm{P} \geq 0.05)$ in G5 and G6 in relation to group treated with $A F$ alone $(\mathrm{G} 4)$. The relative weight $\%$ of liver of broilers was increased significantly $(\mathrm{P} \leq 0.05)$ in $\mathrm{G} 4$ (AF), G5 and G6, and increased non-significantly ( $\mathrm{P} \geq 0.05)$ in $\mathrm{G} 2, \mathrm{G} 3$ and $\mathrm{G} 7$ when compared to control. On the other hand, the liver's relative weight decreased significantly $(P \leq 0.05)$ in $G 7$ and non-significantly ( $P \geq 0.05)$ in $G 5$ and G6 in relation to group treated with AF alone (G4) despite, there are no significant difference (P $\geq 0.05)$ between G5 and G6.

Also, the data are shown in tables 1 and table 2 indicated that relative weight $\%$ of gizzard, abdominal fat, heart, proventriculus, pancreas, bursa of Fabricius, thymus and spleen showed no significant difference among different experimental groups. Data also showed an enhancement in immune organ weights; bursa of Fabricius, thymus and spleen weight showed a numerical increase in G2 and G3, while showed a numerical decrease in G4 (AF) and in all aflatoxicated and treated groups (G5, G6 and G7) when compared to control. Also, they showed a numerical increase in G5, G6 and G7 in relation to group treated with AF alone (G4).

\section{Determination of biochemical changes}

The data obtained in table 3 revealed that ALT, AST, GGT, ALP and bilirubin were non-significantly (P $\geq 0.05)$ affected at 2, 3, 4 and 5 weeks among different experimental groups. But at $5^{\text {th }}$ week, liver enzymes; ALT, AST, GGT and ALP showed a numerical decrease in G2 and G3, while a numerical increase in G4 (AF) and in aflatoxicated and treated groups (G5, G6 and G7) when compared to control. Also, ALT, AST, GGT and ALP enzymes showed a numerical decrease in G5, G6 and G7 in relation to group treated with AF alone (G4). The present results in table 4 imply that creatinine, uric acid, total cholesterol, triacylglycerol, HDL-C and LDL-C were non-significantly ( $\mathrm{P} \geq 0.05$ ) affected at 2, 3, 4 and 5 weeks among different experimental groups. But at $5^{\text {th }}$ week, renal functions (creatinine and uric acid), and lipid profile (total cholesterol, triacylglycerol, HDL-C and LDL-C) showed a numerical decrease in G2 and G3, while a numerical increase in G4 (AF) and in aflatoxicated and treated groups (G5, G6 and G7) when compared to control. Also, creatinine, uric acid, total cholesterol, triacylglycerol, HDL-C and LDL-C showed a numerical decrease in G5, G6 and G7 in relation to group treated with AF alone (G4). 
Table 1. Experimental results regarding dressing percentage and average relative weights $(\%)$ for small intestine, liver, gizzard and abdominal fat of all broiler groups at fifth week

\begin{tabular}{lccccc}
\hline Group/ $\mathrm{n}=30$ & Dressing \% & Small intestine & Liver & Gizzard & Abdominal fat \\
\hline G1 (basal diet/gm) & $58.3 \pm 0.01^{\mathrm{a}}$ & $15.5 \pm 0.6^{\mathrm{ab}}$ & $3.29 \pm 0.8^{\mathrm{c}}$ & $4.72 \pm 1.1^{\mathrm{a}}$ & $1.32 \pm 0.04^{\mathrm{a}}$ \\
G2 (2\% DPS) & $61.2 \pm 0.01^{\mathrm{a}}$ & $15.9 \pm 0.9^{\mathrm{ab}}$ & $4.3 \pm 0.4^{\mathrm{bc}}$ & $5.51 \pm 0.4^{\mathrm{a}}$ & $1.86 \pm 0.06^{\mathrm{a}}$ \\
G3 (4\% DPS) & $59.6 \pm 0.01^{\mathrm{a}}$ & $16.6 \pm 0.9^{\mathrm{a}}$ & $4.2 \pm 0.2^{\mathrm{bc}}$ & $5.61 \pm 0.3^{\mathrm{a}}$ & $1.53 \pm 0.4^{\mathrm{a}}$ \\
G4 (AF) $\mu \mathrm{g} / \mathrm{kg}$ feed & $50.3 \pm 0.01^{\mathrm{a}}$ & $11.1 \pm 1.9^{\mathrm{c}}$ & $5.21 \pm 0.4^{\mathrm{a}}$ & $4.10 \pm 0.2^{\mathrm{a}}$ & $1.31 \pm 0.1^{\mathrm{a}}$ \\
G5 (AF+HSCAS) & $55.4 \pm 0.01^{\mathrm{a}}$ & $13.9 \pm 0.9^{\mathrm{bc}}$ & $4.8 \pm 0.5^{\mathrm{ab}}$ & $4.21 \pm 0.1^{\mathrm{a}}$ & $1.14 \pm 0.12^{\mathrm{a}}$ \\
G6 (AF+2\% DPS) & $57.2 \pm 0.01^{\mathrm{a}}$ & $13.9 \pm 0.6^{\mathrm{bc}}$ & $4.7 \pm 0.3^{\mathrm{ab}}$ & $4.42 \pm 0.3^{\mathrm{a}}$ & $1.33 \pm 0.13^{\mathrm{a}}$ \\
G7 (AF+4\% DPS) & $53.9 \pm 0.01^{\mathrm{a}}$ & $15 \pm 0.3^{\mathrm{ab}}$ & $4.4 \pm 0.3^{\mathrm{bc}}$ & $4.51 \pm 0.2^{\mathrm{a}}$ & $1.2 \pm 0.1^{\mathrm{a}}$ \\
\hline
\end{tabular}

G: group. DPS: date pits ( 2 or $4 \%)$. AF: aflatoxin (100 $\mu \mathrm{g} / \mathrm{kg}$ feed). HSCAS: hydrated sodium calcium aluminosilicate $(0.3 \%)$. Means within the same column under the same category carry different superscripts are significantly different $(\mathrm{P} \leq 0.05)$. $\mathrm{n}=$ number of chicks/ group. gm: gram. $\mu \mathrm{g} / \mathrm{kg}$ : microgram/ kilogram. Values are expressed as means \pm SE.

Table 2. Experimental results regarding average relative weights (\%) for heart, proventriculus, pancreas and lymphoid organs (bursa of Fabricius, thymus and spleen) of all broiler groups at fifth week

\begin{tabular}{|c|c|c|c|c|c|c|}
\hline Group $/ \mathrm{n}=30$ & Heart & Proventriculus & Pancreas & Bursa & Thymus & Spleen \\
\hline G1 (basal diet/gm) & $0.9 \pm 0.15^{\mathrm{a}}$ & $0.65 \pm 0.1^{\mathrm{a}}$ & $0.62 \pm 0.1^{\mathrm{a}}$ & $0.22 \pm 0.01^{\mathrm{a}}$ & $0.23 \pm 0.05^{\mathrm{a}}$ & $0.17 \pm 0.04^{\mathrm{a}}$ \\
\hline G2 (2\%DPS) & $1.0 \pm 0.08^{\mathrm{a}}$ & $0.7 \pm 0.05^{\mathrm{a}}$ & $0.6 \pm 0.04^{\mathrm{a}}$ & $0.24 \pm 0.0^{\mathrm{a}}$ & $0.3 \pm 0.02^{\mathrm{a}}$ & $0.23 \pm 0.02^{\mathrm{a}}$ \\
\hline G3 (4\%DPS) & $1.0 \pm 0.06^{\mathrm{a}}$ & $0.7 \pm 0.04^{\mathrm{a}}$ & $0.7 \pm 0.06^{\mathrm{a}}$ & $0.24 \pm 0.01^{\mathrm{a}}$ & $0.25 \pm 0.03^{\mathrm{a}}$ & $0.19 \pm 0.02^{\mathrm{a}}$ \\
\hline $\mathrm{G} 4(\mathrm{AF}) \mu \mathrm{g} / \mathrm{kg}$ & $1.1 \pm 0.05^{\mathrm{a}}$ & $0.61 \pm 0.1^{\mathrm{a}}$ & $0.5 \pm 0.04^{\mathrm{a}}$ & $0.15 \pm 0.0^{\mathrm{a}}$ & $0.1 \pm 0.03^{\mathrm{a}}$ & $0.12 \pm 0.02^{\mathrm{a}}$ \\
\hline G5 (AF+HSCAS) & $1.0 \pm 0.04^{\mathrm{a}}$ & $0.6 \pm 0.07^{\mathrm{a}}$ & $0.6 \pm 0.06^{\mathrm{a}}$ & $0.18 \pm 0.0^{\mathrm{a}}$ & $0.16 \pm 0.02^{\mathrm{a}}$ & $0.14 \pm 0.01^{\mathrm{a}}$ \\
\hline $\mathrm{G} 6(\mathrm{AF}+2 \% \mathrm{DPS})$ & $1.0 \pm 0.06^{\mathrm{a}}$ & $0.7 \pm 0.04^{\mathrm{a}}$ & $0.6 \pm 0.02^{\mathrm{a}}$ & $0.19 \pm 0.01^{\mathrm{a}}$ & $0.19 \pm 0.04^{\mathrm{a}}$ & $0.16 \pm 0.03^{\mathrm{a}}$ \\
\hline $\mathrm{G} 7(\mathrm{AF}+4 \% \mathrm{DPS})$ & $0.9 \pm 0.07^{\mathrm{a}}$ & $0.7 \pm 0.03^{\mathrm{a}}$ & $0.7 \pm 0.03^{\mathrm{a}}$ & $0.21 \pm 0.0^{\mathrm{a}}$ & $0.17 \pm 0.02^{\mathrm{a}}$ & $0.15 \pm 0.02^{\mathrm{a}}$ \\
\hline
\end{tabular}

G: group. DPS: date pits (2 or 4\%). AF: aflatoxin (100 $\mu \mathrm{g} / \mathrm{kg}$ feed). HSCAS: hydrated sodium calcium aluminosilicate $(0.3 \%)$. Means within the same column under the same category carry different superscripts are significantly different $(\mathrm{P} \leq 0.05)$. Values are expressed as means $\pm \mathrm{SE}$. $\mathrm{n}=\mathrm{number}$ of chicks/ group. gm: gram; $\mu \mathrm{g} / \mathrm{kg}$ : microgram/ kilogram.

Table 3. Experimental results regarding ALT (U/l), AST (U/l), GGT (U/l), ALP (U/l) and bilirubin (mg/dl) for all broiler groups at second, third, fourth and fifth week

\begin{tabular}{|c|c|c|c|c|c|}
\hline ITEM & Group & $2^{\text {nd }}$ Week & $3^{\text {rd }}$ Week & $4^{\text {th }}$ Week & $5^{\text {th }}$ Week \\
\hline \multirow{7}{*}{$\begin{array}{l}\mathbf{A L T} \\
(\mathbf{U} / \mathbf{l})\end{array}$} & G1 (basal diet) & $9.0 \pm 1.3^{\mathrm{a}}$ & $8.9 \pm 1.0^{\mathrm{a}}$ & $9.20 \pm 1.2^{\mathrm{a}}$ & $9.4 \pm 1.0^{\mathrm{a}}$ \\
\hline & $\mathrm{G} 2(2 \% \mathrm{DPS})$ & $9.0 \pm 1.4^{\mathrm{a}}$ & $8.7 \pm 1.6^{\mathrm{a}}$ & $8.60 \pm 1.5^{\mathrm{a}}$ & $8.0 \pm 1.8^{\mathrm{a}}$ \\
\hline & G3 (4\%DPS) & $8.9 \pm 1.8^{\mathrm{a}}$ & $8.8 \pm 1.1^{\mathrm{a}}$ & $8.75 \pm 1.7^{\mathrm{a}}$ & $8.4 \pm 2.3^{\mathrm{a}}$ \\
\hline & $\mathrm{G} 4(\mathrm{AF})$ & $9.1 \pm 1.3^{\mathrm{a}}$ & $9.5 \pm 1.2^{\mathrm{a}}$ & $10.20 \pm 1^{\mathrm{a}}$ & $11.5 \pm 2.1^{\mathrm{a}}$ \\
\hline & G5 (AF+HSCAS) & $9.0 \pm 2.3^{\mathrm{a}}$ & $9.0 \pm 1.3^{\mathrm{a}}$ & $9.50 \pm 2.4^{\mathrm{a}}$ & $9.8 \pm 1.8^{\mathrm{a}}$ \\
\hline & G6 (AF+2\%DPS) & $9.2 \pm 2.5^{\mathrm{a}}$ & $9.3 \pm 2.0^{\mathrm{a}}$ & $9.70 \pm 1.4^{\mathrm{a}}$ & $10 \pm 1.5^{\mathrm{a}}$ \\
\hline & G7 (AF+4\%DPS) & $9.1 \pm 1.0^{\mathrm{a}}$ & $9.4 \pm 1.9^{\mathrm{a}}$ & $9.98 \pm 1.8^{\mathrm{a}}$ & $10.8 \pm 1.2^{\mathrm{a}}$ \\
\hline \multirow{7}{*}{$\begin{array}{l}\text { AST } \\
(\mathbf{U} / \mathbf{l})\end{array}$} & G1 (basal diet) & $188.5 \pm 11^{\mathrm{a}}$ & $188 \pm 39^{a}$ & $187.6 \pm 20^{\mathrm{a}}$ & $194.4 \pm 24^{\mathrm{a}}$ \\
\hline & G2 (2\%DPS) & $188.2 \pm 24^{\mathrm{a}}$ & $186 \pm 12^{\mathrm{a}}$ & $182.6 \pm 13^{\mathrm{a}}$ & $169.4 \pm 14^{\mathrm{a}}$ \\
\hline & G3 (4\%DPS) & $189.0 \pm 28^{\mathrm{a}}$ & $187 \pm 19^{a}$ & $184.6 \pm 10^{\mathrm{a}}$ & $175.8 \pm 11^{\mathrm{a}}$ \\
\hline & G4 (AF) & $188.8 \pm 17^{\mathrm{a}}$ & $189 \pm 30^{\mathrm{a}}$ & $193.6 \pm 19^{\mathrm{a}}$ & $207.0 \pm 26^{\mathrm{a}}$ \\
\hline & G5 (AF+HSCAS) & $189.8 \pm 33^{\mathrm{a}}$ & $189 \pm 19^{a}$ & $192.6 \pm 16^{\mathrm{a}}$ & $195.2 \pm 25^{\mathrm{a}}$ \\
\hline & G6 (AF+2\%DPS) & $189.4 \pm 9^{\mathrm{a}}$ & $189 \pm 20^{\mathrm{a}}$ & $190.2 \pm 35^{\mathrm{a}}$ & $196.6 \pm 18^{\mathrm{a}}$ \\
\hline & G7 (AF+4\%DPS) & $188.8 \pm 22^{\mathrm{a}}$ & $189 \pm 23^{a}$ & $193.6 \pm 10^{\mathrm{a}}$ & $202.6 \pm 13^{\mathrm{a}}$ \\
\hline \multirow{7}{*}{$\begin{array}{l}\text { GGT } \\
(\mathbf{U} / \mathbf{l})\end{array}$} & G1 (basal diet) & $19.18 \pm 0.2^{\mathrm{a}}$ & $19.6 \pm 3.1^{\mathrm{a}}$ & $20.0 \pm 1.3^{\mathrm{a}}$ & $19.8 \pm 2.5^{\mathrm{a}}$ \\
\hline & G2 (2\%DPS) & $19.18 \pm 2.2^{\mathrm{a}}$ & $18.2 \pm 2.9^{\mathrm{a}}$ & $15.8 \pm 2.6^{\mathrm{a}}$ & $14.0 \pm 2.0^{\mathrm{a}}$ \\
\hline & G3 (4\%DPS) & $19.14 \pm 3.6^{\mathrm{a}}$ & $18.6 \pm 3.6^{\mathrm{a}}$ & $18.4 \pm 3.4^{\mathrm{a}}$ & $18.2 \pm 3.1^{\mathrm{a}}$ \\
\hline & G4 (AF) & $19.16 \pm 2.0^{\mathrm{a}}$ & $20.8 \pm 2.8^{\mathrm{a}}$ & $22.6 \pm 3.6^{\mathrm{a}}$ & $24.8 \pm 3.2^{\mathrm{a}}$ \\
\hline & G5 (AF+HSCAS) & $19.14 \pm 3.0^{\mathrm{a}}$ & $19.8 \pm 2.6^{\mathrm{a}}$ & $21.6 \pm 2.2^{\mathrm{a}}$ & $21.8 \pm 2.4^{\mathrm{a}}$ \\
\hline & G6 (AF+2\%DPS) & $19.12 \pm 2.8^{\mathrm{a}}$ & $19.6 \pm 3.0^{\mathrm{a}}$ & $20.6 \pm 2.6^{\mathrm{a}}$ & $21.6 \pm 3.6^{\mathrm{a}}$ \\
\hline & G7 (AF+4\%DPS) & $19.18 \pm 0.8^{\mathrm{a}}$ & $20.0 \pm 3.7^{\mathrm{a}}$ & $22.0 \pm 2.5^{\mathrm{a}}$ & $23.0 \pm 2.5^{\mathrm{a}}$ \\
\hline \multirow{7}{*}{$\begin{array}{l}\mathbf{A L P} \\
(\mathbf{U} / \mathbf{l})\end{array}$} & G1 (basal diet) & $5.8 \pm 1.06^{\mathrm{a}}$ & $6.0 \pm 0.94^{\mathrm{a}}$ & $7.0 \pm 1.20^{\mathrm{a}}$ & $7.4 \pm 1.74^{\mathrm{a}}$ \\
\hline & G2 (2\%DPS) & $5.8 \pm 0.96^{\mathrm{a}}$ & $5.6 \pm 0.97^{\mathrm{a}}$ & $5.3 \pm 1.09^{\mathrm{a}}$ & $4.8 \pm 1.59^{\mathrm{a}}$ \\
\hline & G3 (4\%DPS) & $5.8 \pm 1.15^{\mathrm{a}}$ & $5.7 \pm 1.09^{\mathrm{a}}$ & $5.4 \pm 1.37^{\mathrm{a}}$ & $5.0 \pm 1.4^{\mathrm{a}}$ \\
\hline & G4 (AF) & $5.6 \pm 1.20^{\mathrm{a}}$ & $6.2 \pm 1.24^{\mathrm{a}}$ & $8.2 \pm 1.4^{\mathrm{a}}$ & $10 \pm 1.32^{\mathrm{a}}$ \\
\hline & G5 (AF+HSCAS) & $5.8 \pm 1.59^{\mathrm{a}}$ & $6.1 \pm 1.06^{\mathrm{a}}$ & $7.4 \pm 1.24^{\mathrm{a}}$ & $8.4 \pm 1.44^{\mathrm{a}}$ \\
\hline & G6 (AF+2\%DPS) & $5.6 \pm 1.12^{\mathrm{a}}$ & $6.1 \pm 1.49^{\mathrm{a}}$ & $7.2 \pm 1.51^{\mathrm{a}}$ & $8.0 \pm 1.50^{\mathrm{a}}$ \\
\hline & G7 (AF+4\%DPS) & $5.8 \pm 1.01^{\mathrm{a}}$ & $5.83 \pm 1.57^{\mathrm{a}}$ & $7.9 \pm 1.36^{\mathrm{a}}$ & $8.8 \pm 1.19^{a}$ \\
\hline \multirow{7}{*}{$\begin{array}{l}\text { Bilirubin } \\
\text { (mg/dl) }\end{array}$} & G1 (basal diet) & $0.14 \pm 0.01^{\mathrm{a}}$ & $0.14 \pm 0.02^{\mathrm{a}}$ & $0.15 \pm 0.02^{\mathrm{a}}$ & $0.15 \pm 0.02^{\mathrm{a}}$ \\
\hline & G2 (2\%DPS) & $0.14 \pm 0.02^{\mathrm{a}}$ & $0.14 \pm 0.01^{\mathrm{a}}$ & $0.14 \pm 0.01^{\mathrm{a}}$ & $0.13 \pm 0.02^{\mathrm{a}}$ \\
\hline & G3 (4\%DPS) & $0.14 \pm 0.01^{\mathrm{a}}$ & $0.14 \pm 0.02^{\mathrm{a}}$ & $0.14 \pm 0.02^{\mathrm{a}}$ & $0.14 \pm 0.01^{\mathrm{a}}$ \\
\hline & $\mathrm{G} 4(\mathrm{AF})$ & $0.15 \pm 0.02^{\mathrm{a}}$ & $0.15 \pm 0.02^{\mathrm{a}}$ & $0.15 \pm 0.01^{\mathrm{a}}$ & $0.16 \pm 0.02^{\mathrm{a}}$ \\
\hline & G5 (AF+HSCAS) & $0.14 \pm 0.02^{\mathrm{a}}$ & $0.14 \pm 0.01^{\mathrm{a}}$ & $0.15 \pm 0.02^{\mathrm{a}}$ & $0.15 \pm 0.01^{\mathrm{a}}$ \\
\hline & G6 (AF+2\%DPS) & $0.14 \pm 0.01^{\mathrm{a}}$ & $0.14 \pm 0.02^{\mathrm{a}}$ & $0.15 \pm 0.01^{\mathrm{a}}$ & $0.15 \pm 0.02^{\mathrm{a}}$ \\
\hline & G7 (AF+4\%DPS) & $0.15 \pm 0.02^{\mathrm{a}}$ & $0.15 \pm 0.02^{\mathrm{a}}$ & $0.15 \pm 0.01^{\mathrm{a}}$ & $0.15 \pm 0.02^{\mathrm{a}}$ \\
\hline
\end{tabular}

G: group. DPS: date pits ( 2 or $4 \%)$. AF: aflatoxin (100 $\mu \mathrm{g} / \mathrm{kg}$ feed). HSCAS: hydrated sodium calcium aluminosilicate (0.3\%). ALT: Alanine amino transferase. AST: Aspartate transferase. GGT: Gamma glutamyl transferase. ALP: Alkaline phosphatase. U/l: units per liter. (mg /dl): milligrams per deciliter. Means within the same column under the same category carry different superscripts are significantly different $(\mathrm{P} \leq 0.05)$. Values are expressed as means $\pm \mathrm{SE}$. 
Table 4. Experimental results regarding serum creatinine $(\mathrm{mg} / \mathrm{dl})$, uric acid $(\mathrm{mg} / \mathrm{dl})$, total cholesterol $(\mathrm{mg} / \mathrm{dl})$, triacylglycerol $(\mathrm{mg} / \mathrm{dl})$, high density lipoprotein $(\mathrm{mg} / \mathrm{dl})$ and low density lipoprotein $(\mathrm{mg} / \mathrm{dl})$ for all broiler groups at second, third, fourth and fifth week

\begin{tabular}{|c|c|c|c|c|c|}
\hline ITEM & Group & $2^{\text {nd }}$ Week & $3^{\text {rd }}$ Week & $4^{\text {th }}$ Week & $5^{\text {th }}$ Week \\
\hline \multirow{7}{*}{$\begin{array}{l}\text { Creatinine } \\
(\mathrm{mg} / \mathrm{dl})\end{array}$} & G1 (basal diet) & $0.20 \pm 0.02^{\mathrm{a}}$ & $0.23 \pm 0.03^{\mathrm{a}}$ & $0.24 \pm 0.06^{\mathrm{a}}$ & $0.25 \pm 0.05^{\mathrm{a}}$ \\
\hline & G2 (2\%DPS) & $0.21 \pm 0.04^{\mathrm{a}}$ & $0.21 \pm 0.03^{\mathrm{a}}$ & $0.19 \pm 0.04^{\mathrm{a}}$ & $0.18 \pm 0.04^{\mathrm{a}}$ \\
\hline & G3 (4\%DPS) & $0.26 \pm 0.06^{\mathrm{a}}$ & $0.24 \pm 0.05^{\mathrm{a}}$ & $0.20 \pm 0.04^{\mathrm{a}}$ & $0.19 \pm 0.02^{\mathrm{a}}$ \\
\hline & G4 (AF) & $0.20 \pm 0.03^{\mathrm{a}}$ & $0.25 \pm 0.04^{\mathrm{a}}$ & $0.27 \pm 0.05^{\mathrm{a}}$ & $0.30 \pm 0.04^{\mathrm{a}}$ \\
\hline & G5 (AF+HSCAS) & $0.22 \pm 0.05^{\mathrm{a}}$ & $0.24 \pm 0.03^{\mathrm{a}}$ & $0.25 \pm 0.05^{\mathrm{a}}$ & $0.26 \pm 0.06^{\mathrm{a}}$ \\
\hline & $\mathrm{G} 6(\mathrm{AF}+2 \% \mathrm{DPS})$ & $0.20 \pm 0.03^{\mathrm{a}}$ & $0.24 \pm 0.05^{\mathrm{a}}$ & $0.24 \pm 0.06^{\mathrm{a}}$ & $0.27 \pm 0.07^{\mathrm{a}}$ \\
\hline & G7 (AF+4\%DPS) & $0.21 \pm 0.02^{\mathrm{a}}$ & $0.25 \pm 0.05^{\mathrm{a}}$ & $0.26 \pm 0.03^{\mathrm{a}}$ & $0.28 \pm 0.04^{\mathrm{a}}$ \\
\hline \multirow{7}{*}{$\begin{array}{l}\text { Uric acid } \\
(\mathrm{mg} / \mathrm{dl})\end{array}$} & G1 (basal diet) & $8.34 \pm 0.54^{\mathrm{a}}$ & $8.50 \pm 0.48^{\mathrm{a}}$ & $8.84 \pm 1.48^{\mathrm{a}}$ & $8.92 \pm 0.99^{\mathrm{a}}$ \\
\hline & G2 (2\%DPS) & $8.11 \pm 0.75^{\mathrm{a}}$ & $8.06 \pm 0.64^{\mathrm{a}}$ & $7.70 \pm 0.95^{\mathrm{a}}$ & $7.00 \pm 1.52^{\mathrm{a}}$ \\
\hline & G3 (4\%DPS) & $8.16 \pm 1.32^{\mathrm{a}}$ & $8.04 \pm 0.49^{\mathrm{a}}$ & $7.90 \pm 0.82^{\mathrm{a}}$ & $7.74 \pm 0.57^{\mathrm{a}}$ \\
\hline & $\mathrm{G} 4(\mathrm{AF})$ & $8.46 \pm 0.70^{\mathrm{a}}$ & $8.92 \pm 1.00^{\mathrm{a}}$ & $9.40 \pm 1.07^{\mathrm{a}}$ & $9.86 \pm 1.67^{\mathrm{a}}$ \\
\hline & G5 (AF+HSCAS) & $8.21 \pm 0.73^{\mathrm{a}}$ & $8.64 \pm 1.19^{\mathrm{a}}$ & $8.92 \pm 1.73^{\mathrm{a}}$ & $9.42 \pm 1.0^{\mathrm{a}}$ \\
\hline & G6 (AF+2\%DPS) & $8.36 \pm 0.46^{\mathrm{a}}$ & $8.60 \pm 0.80^{\mathrm{a}}$ & $8.82 \pm 1.49^{\mathrm{a}}$ & $9.28 \pm 1.38^{\mathrm{a}}$ \\
\hline & G7 (AF+4\%DPS) & $8.38 \pm 0.43^{\mathrm{a}}$ & $8.86 \pm 0.71^{\mathrm{a}}$ & $9.02 \pm 0.85^{\mathrm{a}}$ & $9.64 \pm 0.38^{\mathrm{a}}$ \\
\hline \multirow{7}{*}{$\begin{array}{l}\text { S.CH } \\
\text { (mg/dl) }\end{array}$} & G1 (basal diet) & $137 \pm 16.7^{\mathrm{a}}$ & $136.6 \pm 11^{\mathrm{a}}$ & $135.2 \pm 16^{\mathrm{a}}$ & $134.6 \pm 13^{\mathrm{a}}$ \\
\hline & G2 (2\%DPS) & $136 \pm 11.3^{\mathrm{a}}$ & $135.4 \pm 17^{\mathrm{a}}$ & $132.4 \pm 18^{\mathrm{a}}$ & $125.2 \pm 13^{\mathrm{a}}$ \\
\hline & G3 (4\%DPS) & $136.2 \pm 17^{\mathrm{a}}$ & $135.8 \pm 22^{\mathrm{a}}$ & $133.4 \pm 18^{\mathrm{a}}$ & $128.2 \pm 4^{\mathrm{a}}$ \\
\hline & G4 (AF) & $137.5 \pm 17^{\mathrm{a}}$ & $138.2 \pm 16^{\mathrm{a}}$ & $142.8 \pm 15^{\mathrm{a}}$ & $145.2 \pm 8^{\mathrm{a}}$ \\
\hline & G5 (AF+HSCAS) & $137 \pm 10.3^{\mathrm{a}}$ & $137 \pm 19.1^{\mathrm{a}}$ & $139 \pm 18.5^{\mathrm{a}}$ & $140.4 \pm 13^{\mathrm{a}}$ \\
\hline & $\mathrm{G} 6(\mathrm{AF}+2 \% \mathrm{DPS})$ & $137.1 \pm 16^{\mathrm{a}}$ & $137.9 \pm 17^{\mathrm{a}}$ & $139.5 \pm 17^{\mathrm{a}}$ & $140 \pm 17.6^{\mathrm{a}}$ \\
\hline & G7 (AF+4\%DPS) & $137.20 \pm 8^{\mathrm{a}}$ & $138 \pm 11.8^{\mathrm{a}}$ & $138.4 \pm 11^{\mathrm{a}}$ & $142 \pm 9.79^{\mathrm{a}}$ \\
\hline \multirow{7}{*}{$\begin{array}{l}\text { S.TG } \\
(\mathrm{mg} / \mathrm{dl})\end{array}$} & G1 (basal diet) & $165.0 \pm 24^{\mathrm{a}}$ & $166.6 \pm 25^{\mathrm{a}}$ & $165.2 \pm 7^{\mathrm{a}}$ & $165.2 \pm 26^{\mathrm{a}}$ \\
\hline & G2 (2\%DPS) & $164.6 \pm 26^{\mathrm{a}}$ & $163.4 \pm 26^{\mathrm{a}}$ & $160 \pm 16.4^{\mathrm{a}}$ & $158.2 \pm 17^{\mathrm{a}}$ \\
\hline & G3 (4\%DPS) & $164.4 \pm 14^{\mathrm{a}}$ & $164 \pm 20.9^{\mathrm{a}}$ & $162.2 \pm 22^{\mathrm{a}}$ & $159.8 \pm 19^{\mathrm{a}}$ \\
\hline & $\mathrm{G} 4(\mathrm{AF})$ & $165.6 \pm 15^{\mathrm{a}}$ & $171.4 \pm 17^{\mathrm{a}}$ & $173 \pm 23.7^{\mathrm{a}}$ & $175.2 \pm 18^{\mathrm{a}}$ \\
\hline & G5 (AF+HSCAS) & $165 \pm 10.2^{\mathrm{a}}$ & $167.2 \pm 15^{\mathrm{a}}$ & $167.6 \pm 10^{\mathrm{a}}$ & $169.8 \pm 28^{\mathrm{a}}$ \\
\hline & $\mathrm{G} 6(\mathrm{AF}+2 \% \mathrm{DPS})$ & $165.6 \pm 18^{\mathrm{a}}$ & $166.8 \pm 23^{\mathrm{a}}$ & $167.2 \pm 12^{\mathrm{a}}$ & $168.8 \pm 18^{\mathrm{a}}$ \\
\hline & G7 (AF+4\%DPS) & $165.6 \pm 13^{\mathrm{a}}$ & $166.6 \pm 19^{\mathrm{a}}$ & $168.6 \pm 12^{\mathrm{a}}$ & $171 \pm 16.2^{\mathrm{a}}$ \\
\hline \multirow{7}{*}{$\begin{array}{l}\text { HDL-C } \\
(\mathrm{mg} / \mathrm{dl})\end{array}$} & G1 (basal diet) & $77 \pm 7.28^{\mathrm{a}}$ & $76.0 \pm 6.44^{\mathrm{a}}$ & $74.0 \pm 8.22^{\mathrm{a}}$ & $74.0 \pm 8.11^{\mathrm{a}}$ \\
\hline & G2 (2\%DPS) & $76.0 \pm 5.11^{\mathrm{a}}$ & $75.2 \pm 5.34^{\mathrm{a}}$ & $72.0 \pm 5.44^{\mathrm{a}}$ & $68.0 \pm 8.04^{\mathrm{a}}$ \\
\hline & G3 (4\%DPS) & $76.75 \pm 6.1^{\mathrm{a}}$ & $75.5 \pm 1.18^{\mathrm{a}}$ & $72.9 \pm 5.84^{\mathrm{a}}$ & $69.2 \pm 2.51^{\mathrm{a}}$ \\
\hline & G4 (AF) & $78.2 \pm 2.28^{\mathrm{a}}$ & $76.3 \pm 1.98^{\mathrm{a}}$ & $86.0 \pm 8.13^{\mathrm{a}}$ & $85.4 \pm 4.05^{\mathrm{a}}$ \\
\hline & G5 (AF+HSCAS) & $79.3 \pm 3.58^{\mathrm{a}}$ & $76.0 \pm 1.09^{\mathrm{a}}$ & $76.8 \pm 8.08^{\mathrm{a}}$ & $81.0 \pm 3.31^{\mathrm{a}}$ \\
\hline & $\mathrm{G} 6(\mathrm{AF}+2 \% \mathrm{DPS})$ & $77.8 \pm 3.51^{\mathrm{a}}$ & $76.1 \pm 5.1^{\mathrm{a}}$ & $76.6 \pm 5.55^{\mathrm{a}}$ & $79.3 \pm 4.28^{\mathrm{a}}$ \\
\hline & G7 (AF+4\%DPS) & $78.0 \pm 4.98^{\mathrm{a}}$ & $76.2 \pm 5.83^{\mathrm{a}}$ & $77.3 \pm 4.84^{\mathrm{a}}$ & $83.6 \pm 5.32^{\mathrm{a}}$ \\
\hline \multirow{7}{*}{$\begin{array}{l}\text { LDL-C } \\
\text { (mg/dl) }\end{array}$} & G1 (basal diet) & $32.1 \pm 2.38^{\mathrm{a}}$ & $32.3 \pm 2.61^{\mathrm{a}}$ & $32.0 \pm 2.36^{\mathrm{a}}$ & $31 \pm 1.97^{\mathrm{a}}$ \\
\hline & G2 (2\%DPS) & $32.02 \pm 2.1^{\mathrm{a}}$ & $31.9 \pm 1.0 \mathrm{a}$ & $30.0 \pm 5.47^{\mathrm{a}}$ & $28 \pm 3.96^{\mathrm{a}}$ \\
\hline & G3 (4\%DPS) & $32.1 \pm 3.28^{\mathrm{a}}$ & $32.0 \pm 1.3^{\mathrm{a}}$ & $31.0 \pm 4.7^{\mathrm{a}}$ & $29 \pm 2.81^{\mathrm{a}}$ \\
\hline & $\mathrm{G} 4(\mathrm{AF})$ & $32.3 \pm 2.7^{\mathrm{a}}$ & $32.4 \pm 3.57^{\mathrm{a}}$ & $34.5 \pm 2.24^{\mathrm{a}}$ & $37 \pm 2.3^{\mathrm{a}}$ \\
\hline & G5 (AF+HSCAS) & $32.2 \pm 1.59^{\mathrm{a}}$ & $32.3 \pm 3.43^{\mathrm{a}}$ & $33 \pm 2.98^{\mathrm{a}}$ & $34 \pm 2.94^{\mathrm{a}}$ \\
\hline & $\mathrm{G} 6(\mathrm{AF}+2 \% \mathrm{DPS})$ & $32.2 \pm 1.68^{\mathrm{a}}$ & $32.3 \pm 2.5^{\mathrm{a}}$ & $32.7 \pm 4.51^{\mathrm{a}}$ & $33.3 \pm 4.72^{\mathrm{a}}$ \\
\hline & G7 (AF+4\%DPS) & $32.3 \pm 2.5^{\mathrm{a}}$ & $32.4 \pm 3.35^{\mathrm{a}}$ & $33.5 \pm 1.0^{\mathrm{a}}$ & $35.5 \pm 2.34^{\mathrm{a}}$ \\
\hline
\end{tabular}

G: group. DPS: date pits (2 or $4 \%)$. AF: aflatoxin $(100 \mu \mathrm{g} / \mathrm{kg}$ feed). HSCAS: hydrated sodium calcium aluminosilicate $(0.3 \%)$. S.CH: Serum cholesterol. S.TG: Serum triglyceride. HDL-C: High density lipoproteins concentration. LDL-C: Low density lipoproteins concentration. (mg /dl): milligrams per deciliter. Means within the same column under the same category carry different superscripts are significantly different (P $\leq 0.05$ ). Values are expressed as means $\pm \mathrm{SE}$.

\section{Histopathological examination of the liver and kidney}

The examination of liver of birds in G1, G2 and G3 showed normal histological appearance and structure (Figures 1,2 and 3 respectively). While the liver of birds of G4 exhibited hemorrhage replaced necrotic hepatocytes (Figure 4a) and focal hepatic necrosis with inflammatory cell infiltration and fatty degeneration of hepatocytes (Figure 4b). Moreover, the examined liver of birds in G5 showed moderate congestion of blood vessels (Figure 5) and in G6 showed mild activation of inflammatory cell infiltration (Figure 6) and finally in G7, moderate inflammatory cells infiltration was seen (Figure 7). 


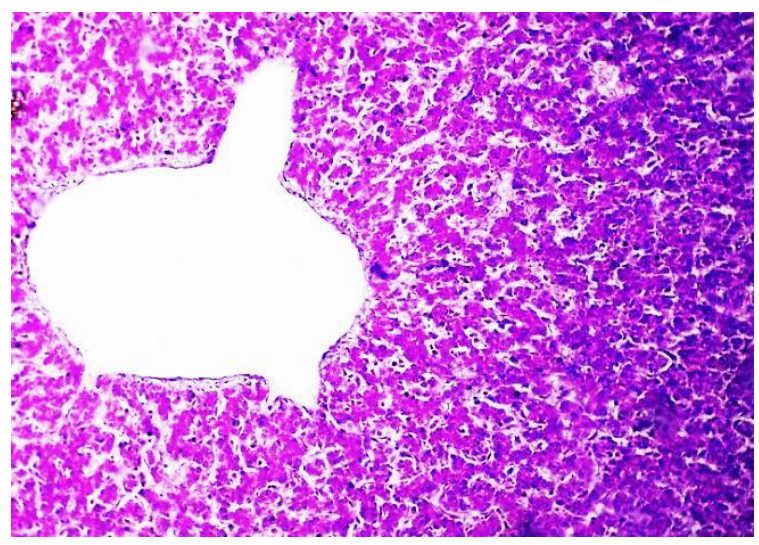

Figure 1. Liver of chicken of G1 (basal diet) at 35 days old showing normal histological structure. H\&E. (x160).

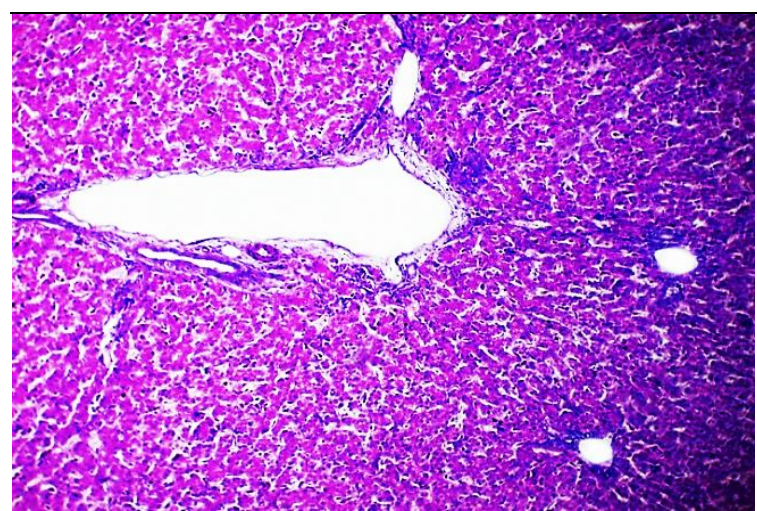

Figure 3. Liver of a chicken of G3 (4\% date pits) at 35 days' old showing normal histological structure. H\&E. (x160).

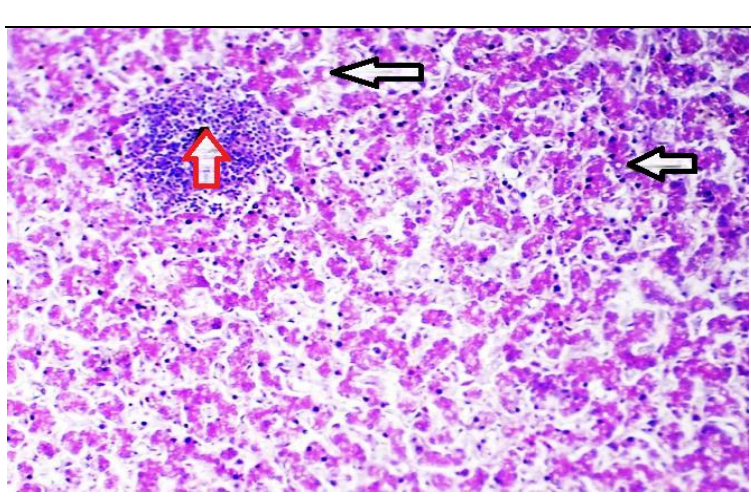

Figure 4b. Liver of a chicken of G4 (AFlatoxin) at 35 days' old showing focal hepatic necrosis with inflammatory cell infiltration (red arrow) and fatty degeneration of hepatocytes (black arrows). H\&E. (x160).

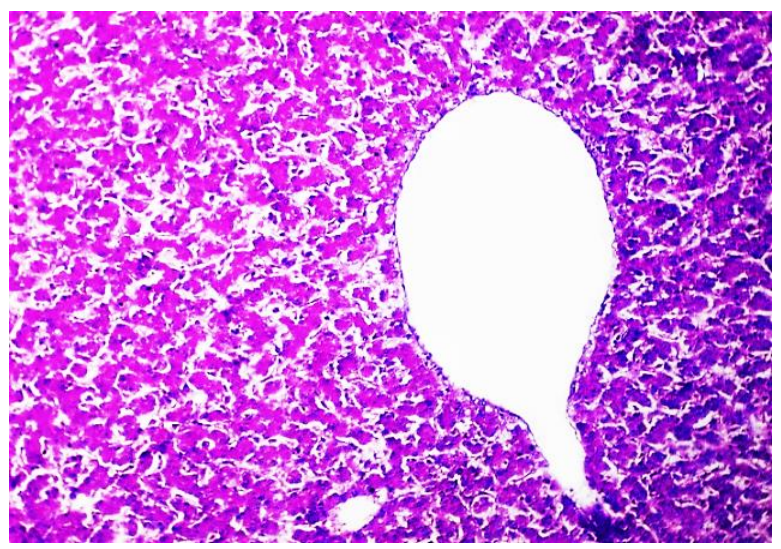

Figure 2. Liver of a chicken of G2 (2\% date pits) at 35 days' old showing normal histological structure. H\&E. (x160).

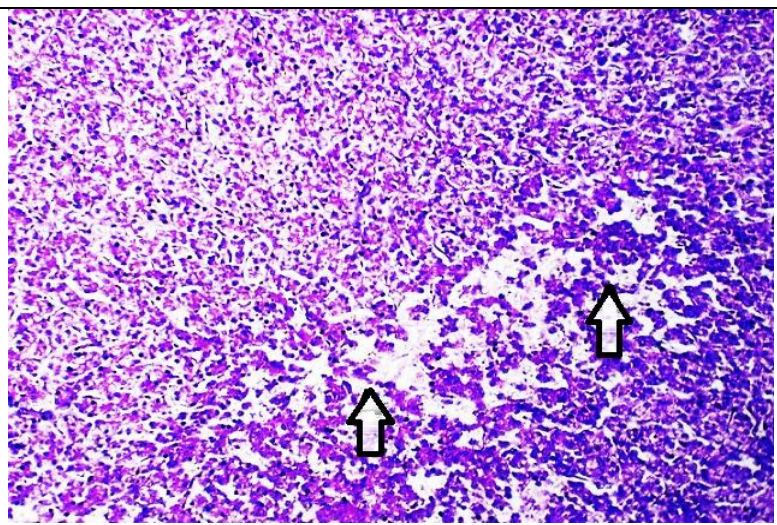

Figure 4a. Liver of a chicken of G4 (AFlatoxin) at 35 days' old showing hemorrhage replaced necrotic hepatocytes (arrows). H\&E. (x160).

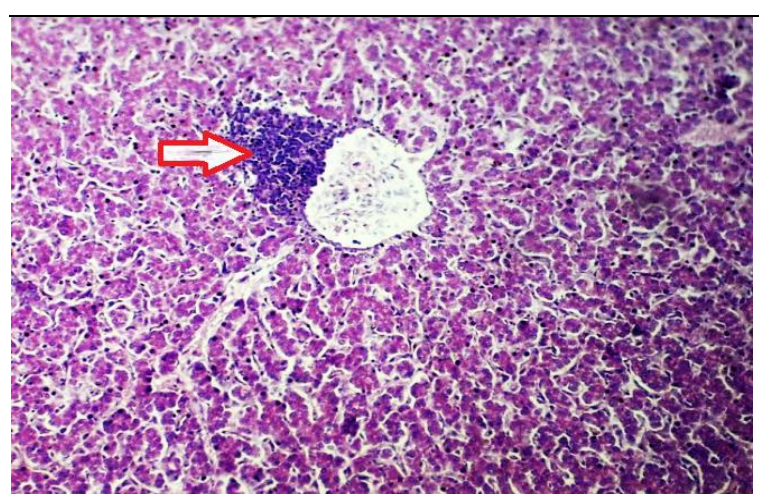

Figure 6. Liver of a chicken of G6 (AFlatoxin $+2 \%$ date pits) at 35 days' old showing mild inflammatory cell infiltration (arrow). H\&E. (x160).

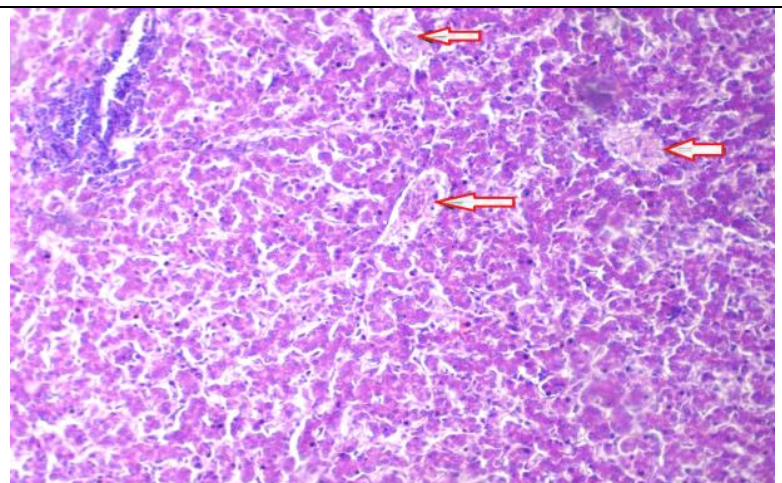

Figure 5. Liver of a chicken of G5 (AFlatoxin+ Hydrated sodium and calcium aluminum Silicates) at 35 days' old showing moderate congestion of blood vessels (arrows). H\&E. (x160).

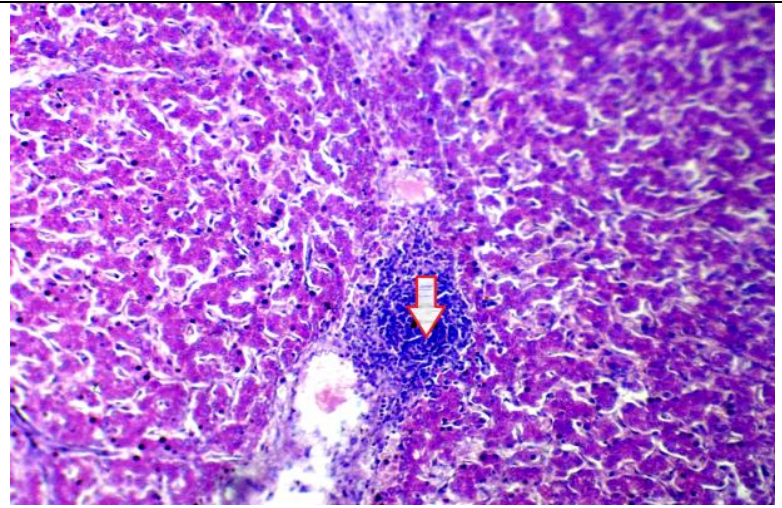

Figure 7. Liver of a chicken of G7 (AFlatoxin+4\% date pits) at 35 days' old showing moderate inflammatory cells infiltration (arrow) in portal area. H\&E. (x160). 


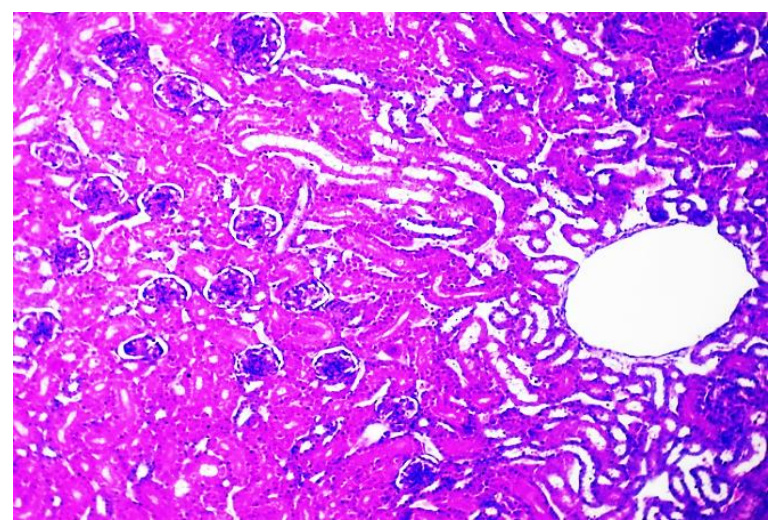

Figure 8. Kidney of a chicken of G1 (basal diet) at 35 days old showing normal histological structure. H\&E. (x160).

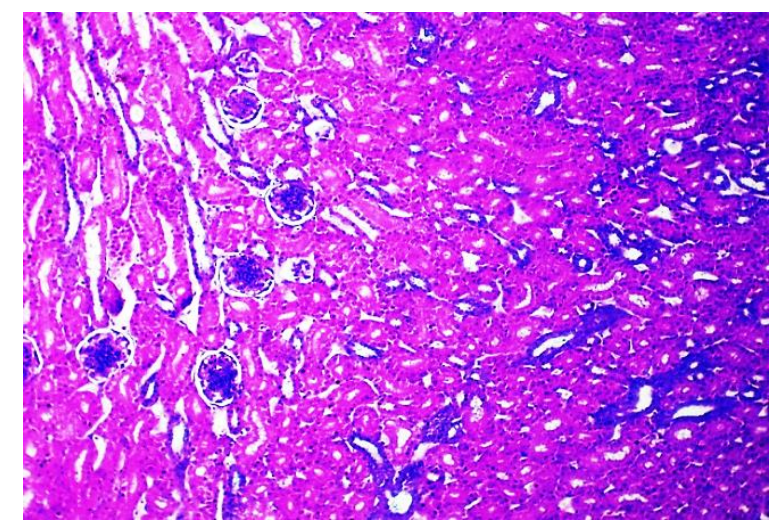

Figure 9. Kidney of a chicken of G2 (2\% date pits) at 35 days' old showing normal histological structure. H\&E. (x160).

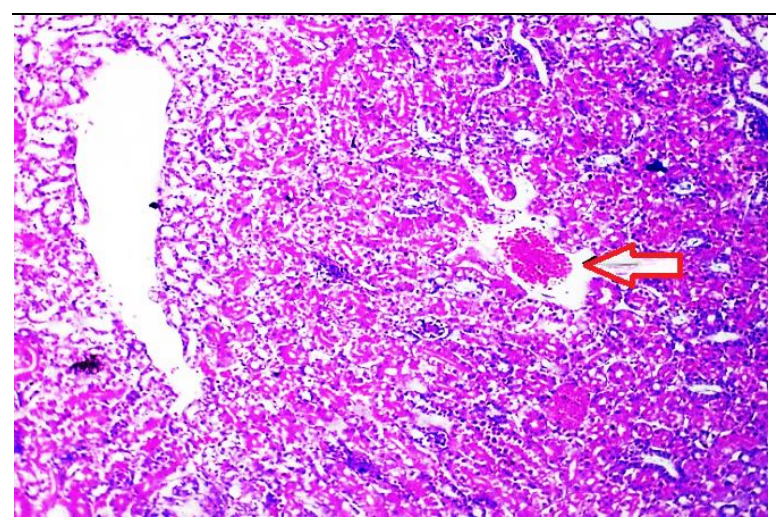

Figure 10. Kidney of a chicken of G3 (4\% date pits) at 35 days' old showing normal histological structure with mild congestion of blood vessel (arrow). H\&E. (x160).

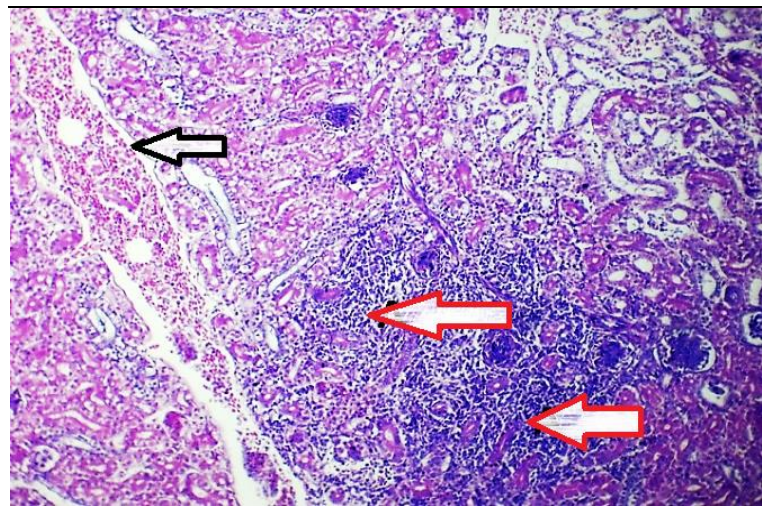

Figure 11b. Kidney of a chicken of G4 (AFlatoxin) at 35 days' old showing interstitial nephritis with mononuclear cell infiltrations (red arrow) and congestion of blood vessel (black arrow). H\&E.

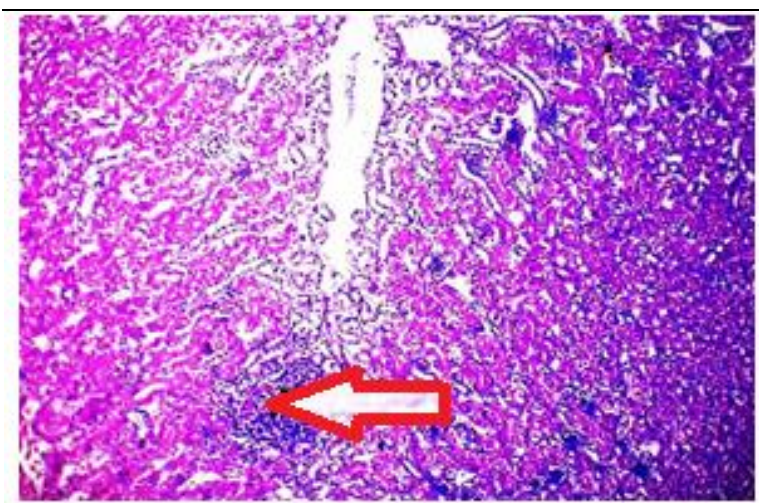

Figure 13. Kidney of a chicken of G6 (AFlatoxin+2\% date pits) at 35 days' old showing focal interstitial nephritis with mononuclear cell infiltrations (arrow). H\&E. (x160).

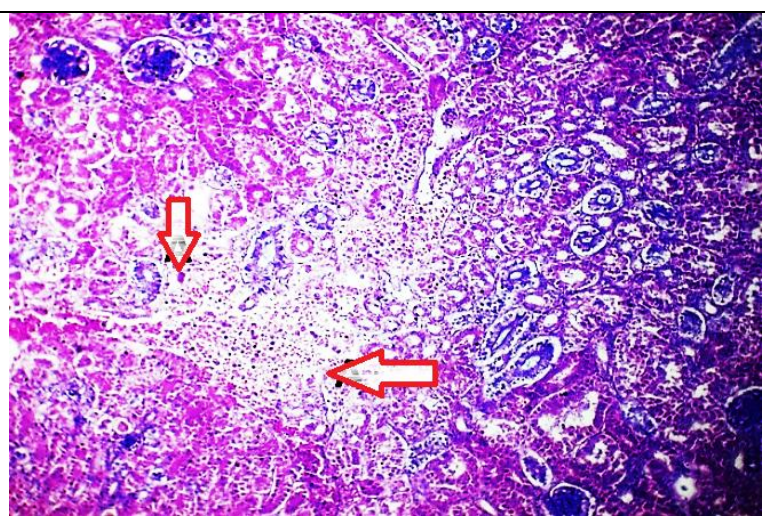

Figure 11a: Kidney of a chicken of G4 (AFlatoxin) at 35 days' old showing hemorrhage (arrow). H\&E. (x160)

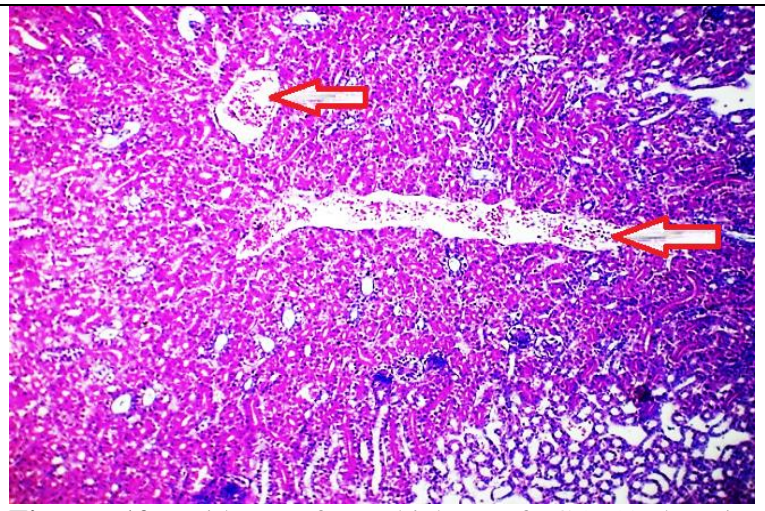

Figure 12. Kidney of a chicken of G5 (AFlatoxin+ Hydrated sodium and calcium aluminum Silicates) at 35 days' old showing mild to moderate congestion of blood vessel (arrows). H\&E. (x160).

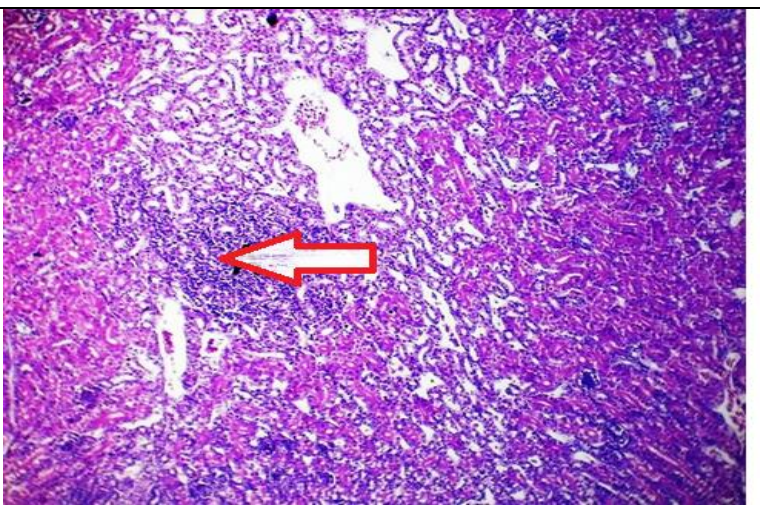

Figure 14. Kidney of a chicken of G7 (AFlatoxin+4\% date pits) at 35 days' old showing focal interstitial nephritis with mononuclear cell infiltrations (arrow). H\&E. (x160). 
The kidney of birds in G1 and G2 exhibited normal histological structure of glomeruli and renal tubule (Figures 8 and 9, respectively), while, in the sacrificed birds of G3 showed normal histological structure with mild congestion of blood vessel (Figure 10). The kidney of birds in G4 showed hemorrhage, where erythrocytes escape from the blood vessel beside interstitial nephritis with mononuclear cell infiltrations and congestion of blood vessel (Figures 11a and $11 \mathrm{~b}$ respectively). Moreover, the kidney of the examined birds in G5 showed mild to moderate congestion of blood vessel (Figure 12), while, the microscopical findings of the kidney of birds in G6 and G7 showed focal interstitial nephritis with mononuclear cell infiltrations (Figures 13 and 14, respectively). Finally, no significant toxic microscopic lesions and normal histology were evident in liver and kidney sections of birds in G1 (control) or G2, G3 fed DPS $2 \%$ and $4 \%$ respectively and all these 3 groups had no mortalities.

\section{DISCUSSION}

Many feed additives had the ability to relieve aflatoxicosis but few researches are carried out on incorporation of date palm seed powder into the diet for poultry production. Therefore, this investigation provides some information to solve the problems of aflatoxicosis in poultry industry by introducing DPS in to the broiler's diets.

In this study, just the relative weight of both liver and small intestine of chickens showed significant differences. The liver's relative weight increased significantly in chickens fed Aflatoxin alone while decreased in chickens fed aflatoxin and treated with $2 \%$ and $4 \%$ date pits, which indicated that supplementation of date pits ameliorate the toxic effect of aflatoxin and utilized properly to improve growth and carcass traits. Bovo et al. (2015) reported that prolonged exposure to dietary AFB1 raised the relative weight of the liver. The relative liver weight is significantly increased by different levels of aflatoxin compared with any other organ, and this enhancement could due to an inhibition of lipid transport and lipid accumulation in the liver (Huff et al., 1986) or could be primarily attributed to necrosis, bile duct proliferation, fat infiltration, and enlarged liver cells (Yunus et al., 2011). Contraindicated results were noticed in previous reports, in which the liver weight of broilers did not change when the dietary AF level was lower as $90.2 \mu \mathrm{g} / \mathrm{kg}$ of diet but this might be due to the shorter duration of aflatoxin supplementation than our experiment (Fan et al., 2015).

The toxic effects of AFlatoxin on blood biochemical parameters were exhibited only at fifth week of ag in chickens fed diet containing aflatoxin alone where increased the concentrations of liver enzymes (ALT, AST, GGT and ALP), kidney functions (creatinine and uric acid) and lipid profile (total cholesterol, triacylglycerol, HDL-C and LDL-C). This may be due to the prolonged hepatic and renal damage and release of enzymes into the blood stream of chickens received AF resulting in chronic venous congestion with circulatory and degenerative changes in most of the body tissues (Jindal et al., 1994). The results matched with the previous findings in which aflatoxin treatment increased ALT, AST, GGT enzymes and served as markers of liver and kidney damage/dysfunction, which promoted the release of these aminotransferases from hepatocytes into the blood stream, indicating liver inflammation, lesions or obstruction of the biliary tract (Neeff et al., 2013; Uyar et al., 2016).

The numerical increase of kidney functions (creatinine and uric acid) in experimental birds that consumed diet contaminated with (100 ppb aflatoxin) at fifth week, as reported previously, may be due to the disturbed transportation function of epithelial cells in collecting tubules and diffuse impairment of proximal tubules' function (Hochleithner, 1994; Umar et al., 2012). Uric acid is the primary end product of protein metabolism in birds. It is synthesized in the liver and excreted through the kidney tubules. Increased blood uric acid and creatinine in broiler chickens receiving 50, 150 and $300 \mathrm{mg} / \mathrm{kg}$ aflatoxin in feed over 42 days were observed previously by George et al. (2006).

The addition of DPS to diet containing $100 \mathrm{ppb}$ AF induced differences in serum parameters at $5^{\text {th }}$ week of age; the liver enzymes (ALT, AST, GGT and ALP), kidney functions (creatinine and uric acid) and lipid profile (total cholesterol, triacylglycerol, HDL-C and LDL-C) showed a numerical decrease in G6 (DPS2\%+AF) and G7 (DPS4\%+AF) comparing with G4 treated with AF alone, indicating the hepatorenal protective activity of DPS against AF. The decrease in serum parameters may have been due to decreased release of tissue specific enzymes and other intracellular proteins which secondary to oxidative stress during metabolism. The mechanism by which DPS induces its protective activity is not clear but possibly due to the antioxidant effect and the content of vitamin C in DPS (0.137\%) which may play a role in hepatoprotection (Burtis and Ashwood, 2001). Moreover, Vayalil (2002) reported that date fruit has antioxidant and antimutagenic activity and this implicated the presence of compounds with potent free-radicalscavenging activity.

The present results were according to the findings of Al-Ghasham et al. (2008) who reported the liver enzymes (ALT and AST) in AFB1 $(50 \mu \mathrm{g} / \mathrm{kg} \mathrm{BW})$ treated group (half dose to our work) were significantly higher than in the control group, while in the AFB1 $(50 \mu \mathrm{g} / \mathrm{kg} \mathrm{BW})$ and date group, the plasma levels of liver function enzymes (ALT and AST), creatinine and urea were significantly lower than the AFB1 group.

Histopathologically, supplementation of $100 \mathrm{ppb}$ aflatoxin in G4 which had severe liver and kidney lesions as indicative for aflatoxicosis exhibited through hemorrhage and focal hepatic necrosis with inflammatory cell infiltration and fatty degeneration of hepatocytes which may be due to disturbance of oxidant/antioxidant balance system and kidney 
as congestion of renal blood vessels, interstitial nephritis with mononuclear cell infiltrations. Also, previous researches which recorded the toxic effect of aflatoxins in liver (Gholami-Ahangaran et al., 2016 and Tessari et al., 2006) and kidney (Mohamed and Mohamed, 2009). The inclusion of DPS to diet contains $100 \mathrm{ppb}$ AF as in G6 and G7 reduced the severity of pathological changes. Although it was not only in liver but also in kidney and it seemed that the effect of mannanoligosacharides (MOS) in DPS on AF toxicity was not only related to the binding capacity with AF and also these MOS could prevent the colonization of opportunistic bacterial pathogens in the gastrointestinal tract (Olsen, 1995). Moreover, Al-Ghasham et al. (2008) reported that the liver and kidney of rats treated with AFB1 and Date, showed nearly normal and the mild changes were just vacuolation of hepatocytes, congestion and few mononuclear cells infiltration. The beneficial effect of date pits appeared in histopathology of both liver and kidney might be due to its antioxidant role.

Supplementation of HSCAS in the diet containing (100 ppb AF) in G5 showed a numerical decrease in liver enzymes (ALT, AST, GGT and ALP), kidney functions (creatinine and uric acid) and lipid profile (total cholesterol, triacylglycerol, HDL-C and LDL-C) compared with chickens receiving AF alone (G4) at $5^{\text {th }}$ week. HSCAS have been demonstrated to be effective in binding $\mathrm{AF}$ molecules in the gastrointestinal tract, making them unavailable for adsorption and consequently alleviating aflatoxicosis (Phillips et al., 1990 and Kubena et al., 1990). Similar results, increased urea and creatinine as indices of impaired kidney function in aflatoxicosis were reported in chickens and rats, a partly reduction of the toxic effects on kidney function was achieved by adding mycosorbents to contaminated feeds (Yildirim et al., 2011).

However, the addition of HSCAS to diet contains (100 ppb of AF) in G5 couldn't fully improve the organ's histopathology as there were moderate congestion of hepatic blood vessels and mild to moderate congestion of renal blood vessels which indicated that HSCAS didn't completely protect broilers against aflatoxicosis but partially ameliorated its effect which may be attributed to the dose of HSCAS as mentioned previously by Neeff et al. (2013) who reported that HSCAS didn't completely protect broilers against aflatoxicosis, but was effective in reducing aflatoxin residues in liver and kidney of chicks fed $2.5 \mathrm{mg}$ of AFB1/kg of diet during 0-21 days of age. Also, Phillips (1999) reported that the protective effect of HSCAS resulted from the rapid binding capacity of HSCAS to aflatoxins in the gastrointestinal tract of chickens, thus preventing its absorption and normal distribution to the liver. This effect could be increased through higher HSCAS dose supplemented in feed.

As the small intestine's relative weight decreased significantly in chickens fed Aflatoxin alone while increased in chickens fed aflatoxin and treated with $2 \%$ and $4 \%$ date pits, indicating that mannan-oligosaccharides in DPS increased the enteric development. These results agreed with Daneshyar et al. (2014) who reported the increased weight of the small intestine, due to decreased activity of AF in the intestines of chickens that received aflatoxicated diets (300 ppb) and supplemented with $4 \%$ date pits. In addition to, Yunus et al. (2011) who reported that dietary exposure to AFB1 can lower the unit weight (length/weight) of the duodenum and jejunum.

The absence of significant differences in dressing percentage and relative weight of gizzard, abdominal fat, heart, proventriculus, pancreas, Bursa of Fabricius (BF), thymus and spleen among different experimental groups agreed with the findings of researches on aflatoxin supplementation, Del Bianchi et al. (2005) who observed no differences in the relative weights of the analyzed organs (heart, BF, thymus, pancreas and proventriculus) among treatments in broilers fed AFB1 from 21 to 42 days of age. Fan et al. (2015) stated that no significant differences were observed for the weights of organs (heart, liver, spleen, BF and thymus) among all treatments in broilers fed mixed AFB1, B2, G1, G2 from 7 to 42 days of age. Moreover, on DPS supplementation, Zangiabadi and Torki (2010) reported that adding complete waste of $30 \%$ dates only significantly affected the relative gizzard weight but had no significant effect on the other body parts.

Finally, the safety of supplementation of DPS alone to the broiler's diet was recorded in G2 (2\% DPS) and G3 (4\% DPS) as it didn't have any drawback on the measured the biochemical parameters at $5^{\text {th }}$ week These results are in accordance with those of earlier studies which investigated the effect of DPS and stated that there were no significant change in liver function (ALT and AST) and kidney function (Urea and creatinine) as well as metabolic markers (total cholesterol and triglycerides), indicating absence of any adverse effects of feeding date pits on hepatic and renal functions (Kamel et al., 2016).

\section{CONCLUSION}

Addition of date palm (2\% and 4\%) to broiler's diet ameliorated the hazardous effects of aflatoxins and this protection is dose-related as $2 \%$ supplementation gave better protection than the higher dose $4 \%$. So, we advise to use date palm as a feed additive to control aflatoxicosis in poultry farms, avoiding the harmful chemical mycotoxin binders causing appreciable losses in nutritive value and palatability. Concomitantly, further studies on the combinations of date palm and other medicinal plants having protective effect against aflatoxicosis in poultry should be applied. 


\section{DECLARATIONS}

\section{Acknowledgements}

The authors would like to offer special thanking for Desert Research Center, Egypt; Poultry Diseases Dep. As well as Biochemistry Department and poultry diseases Department faculty of veterinary medicine, Damnhour University, Egypt for helpful and support.

\section{Competing interests}

With respect to the research, authorship, and/or publications of this article. The authors declare that they have no competing interests.

\section{Author's contribution}

Dr. Disouky Mourad designed the experiment, article writing and revision, Dr. Kadry Saadek designed the experiment, manuscript writing, commenting and approval, Dr. Ahmed El-Bestawy helped in field study, collected data, laboratory analyses, statistical analysis, tabulation of experimental data and article writing; while, Dr. Ward Masoud helped in experiment application, statistical analysis, manuscript writing. All authors have read and approved the final manuscript.

\section{REFERENCES}

Al-Farsi MA and Lee CY (2011). Usage of date (Phoenix dactylifera L.) seeds in human health and animal feed. Nuts and seeds in health and disease prevention book, chapter 53, pp. 447-452. DOI:10.1016/B978-0-12-375688-6.10053-2.

Al-Ghasham, A., Ata H.S, El-Deep S, Meki AR and Shehada S (2008). Study of protective effect of date and Nigella sativa on aflatoxin B1 toxicity. International journal of health sciences, 2(2): .26-44. PMID:21475486.

Bintvihok A and Kositcharoenkul S (2006). Effect of dietary calcium propionate on performance, hepatic enzyme activities and aflatoxin residues in broilers fed a diet containing low levels of aflatoxin B1. Toxicon, 47 (1): 41-46. DOI: 10.1016/J. toxicon 2005. 09. 009.

Bovo F, Franco LT, Kobashigawa E, Rottinghaus GE, Ledoux DR and Oliveira CAF (2015). Efficacy of beer fermentation residue containing Saccharomyces cerevisiae cells for ameliorating aflatoxicosis in broilers. Poultry science, 94(5): 934-942. DOI: 10.3382 /ps / pev 067.

Burtis CA and Ashwood ER (2001). Tietz's Fundamentals of Clinical Chemistry, Philadelphia: WB Saunders, Elsevier, 11830 westline industrial Drive St. louis, Missouri 63146. BN: 978-0-7216-3865-2. Library of congress control number, 2007921126.

Copley M S, Rose P J, Clapham A, Edwards D N, Horton M C and Evershed R P (2001). Detection of palm fruit lipids in archaeological pottery from Qasr Ibrim, Egyptian Nubia. Proceedings of the Royal Society of London B: Biological Sciences, 268(1467): 593-597. DOI: 10.1098/ rspb. 2000. 1394.

Daneshyar F, Afzali N and Farhangfar H (2014). Effects of different levels of date pits in broilers' feed contaminated with aflatoxin B 1 on broilers' performance and carcass characteristic. African Journal of Biotechnology, 13(1): 185-193. DOI:.10.5897/ AJB 2013. 13132.

Del Bianchi M, Oliveira C A F, Albuquerque R, Guerra J L and Correa B (2005). Effects of prolonged oral administration of aflatoxin B1 and fumonisin B1 in broiler chickens. Poultry Science, 84 (12):1835-1840. DOI: 10. 1093 / ps / 84. 12. 1835.

Drury RAB and Wallington EA (1980). Preparation and Fixation of Tissues in Carleton's Histological Technique, fifth ed. Oxford, Newyork, Oxford University Press.

Edds G T and Bortell R R (1983). Biological effects of aflatoxin in poultry. Southern cooperative series bulletin. Agris.fao.org., SIDALC.NET, G279, G56-61, 1983.

Edrington T, Sarr A, Kubena L, Harvey R and Phillips T (1996). (HSCAS), acidic HSCAS, and activated charcoal reduce urinary excretion of aflatoxin M 1 in turkey poults. Lack of effect by activated charcoal on aflatoxicosis. Toxicology letters, 89 (2): 115122 PMID: 8960153. DOI: 10.1016 / 50378 - 4274 (96) 03795- 2.

Fan Y, Zhao L, Ji C, Li X, Jia R, Xi L, Zhang J and Ma Q (2015). Protective effects of bacillus subtilisansb060 on serum biochemistry, histopathological changes and antioxidant enzyme activities of broilers fed moldy peanut meal naturally contaminated with aflatoxins. Toxins, 7(8): .3330-3343. DOI: 10.3390 / toxins / 7083330

FAO (2010). Food and agriculture organization of the United Nations. Rome: Date Palm cultivation.

George A J, Balachandran C, Murali Manohar B, Dhinakar Raj G and Kirubakaran J J (2006). Pathological changes in broiler chicken exposed to sublethal doses of aflatoxin in broiler chicken. In: Compendium of invited papers and abstracts and souvenir, XIII Indian Association of Veterinary Pathologist Conference, 27-29, Chennai.

Gholami-Ahangaran M, Rangsaz N and Azizi S (2016). Evaluation of turmeric (Curcuma longa) effect on biochemical and pathological parameters of liver and kidney in chicken aflatoxicosis. Pharm biol, 54(5): 780-787. PMID: 26450181. DOI 10.3109 / 13880209.2015.1080731.

Golshan Tafti A, Solaimani Dahdivan N and Yasini Ardakani SA (2017). Physicochemical properties and applications of date seed and its oil. International Food Research Journal, 24(4): 1399-1406.

Hochleithner M (1994). Biochemistries. In: Avian Medicine and Surgery: Principles and Applications. eds B. W. Ritchie, G. J. Harrison \& L. R. Harrison, Wingers Publishing Inc., Lake Worth, FL: 223-245. 
Huff W E, Kubena L F, Harvey R B, Corrier D E and Mollenhauer H H (1986). Progression of aflatoxicosis in broiler chickens. Poultry Science, 65 (10):1891-1899. DOI: 10.3382 / ps. 0651891. PMID: 3797371.

Jalili M, Jinap S and Son R (2011). The effect of chemical treatment on reduction of aflatoxins and ochratoxin A in black and white pepper during washing. Food Additives and Contaminants, 28(4): 485-493. DOI: 10.1080/19440049.2010.551300. PMID: 21416415.

Jindal N, Mahipal SK and Mahajan NK (1994). Effect of hydrated sodium calcium aluminosilicate on prevention of aflatoxicosisin broilers. Indian Journal of Animal Science,63: 649-652.

Kamel ER, Manaa E and Farid AS (2016). The Effects of Dietary Date Pit on the Productive and Economic Efficiency of Japanese Quail. Alexandria Journal for Veterinary Sciences, 51(2): 211-221. DOI: 10.5455/ ajvs.233838.

Kubena LF, Harvey RB, Huff WE, Corrier DE, Phillips TD and Rottinghaus GE (1990). Efficacy of hydrated sodium calcium aluminosilicate to reduce the toxicity of aflatoxin and T-2 toxin. Poultry Science, 69 (7):1078-1086. DOI:10.3382/ ps.0691078. PMID: 2235827.

Mohamed M.A and Mohamed M H (2009). Hemato-biochemical and pathological studies on aflatoxicosis and treatment of broiler chicks in Egypt. Veterinaria Italiana, 45 (2): 323-337. PMID: 20391383.

Navid Shad BJSA (2007). Animal Nutrition (translated). (Sixth edition. Haghshenas publications). 702-700.

Neeff D V, Ledoux D R, Rottinghaus G E, Bermudez A J, Dakovic A, Murarolli R A and Oliveira C A F. 2013. In vitro and in vivo efficacy of a hydrated sodium calcium aluminosilicate to bind and reduce aflatoxin residues in tissues of broiler chicks fed aflatoxin B1. Poultry Science, 92 (1):131-137. DOI: 10.3382/ ps.2012-02510. PMID: 23243239.

NRC (1994). National Research Council: Nutrient requirement of Poultry. National Academy Press, Washington, DC. Pp. DOI: $10.17226 / 2114$.

Olsen R (1995). Mannanoligosaccharides: Experience in commercial turkey production. Biotechnology in the Feed Industry. TP Lyons and KA Jacques. ed. Nottingham University Press, Loughborough, Leics, UK, 389-392.

Phillips T D (1999). Dietary clay in the chemoprevention of aflatoxin-induced disease. Toxicological sciences: an official journal of the Society of Toxicology, 52 (2):118-126. DOI: 10.1093/ toxsci/ 52. Suppl-1.118. PMID: 10630600.

Phillips T D, Clement B A, Kubena L F and Harvey R B (1990). Detection and detoxification of aflatoxins: Prevention of aflatoxicosis and aflatoxin residues with hydrated sodium calcium aluminosilicate. Veterinary and Human Toxicology, 32: 15-19. PMID: 1965459.

Rawal S, Kim JE and Coulombe R (2010). Aflatoxin B1 in poultry: Toxicology, metabolism and prevention. Research in Veterinary Science, 89 (3): 325-331. DOI: 10.1016/ J. rvsc.2010.04.011. PMID: 20462619.

Shotwell OL, Hesseltine CW, Stubblefield RD and Sorenson WG (1966). Production of aflatoxin on rice. Journal of Applied Microbiology, 14(3): 425-428. PMID: 5970829.

Sirisena S, Ng K and Ajlouni S (2015). The Emerging Australian Date Palm Industry: Date Fruit Nutritional and Bioactive Compounds and Valuable Processing By-Products. Comprehensive Reviews in Food Science and Food Safety, 14(6): 813-823. DOI: $10.1111 / 1541-4337.12162$.

Tessari ENC, Oliveira CAF, Cardoso ALSP, Ledoux DR and Rottinghaus GE (2006). Effects of aflatoxin B1 and fumonisin B1 on body weight, antibody titres and histology of broiler chicks. British poultry science, 47(3): 357-364. DOI: 10.1080/ 00071660600756071. PMID: 16787861.

Umar S, Arshad A, Ahmad B and Arshad M (2012). Clinico biochemical and hematological changes in broilers induced by concurrent exposure to aflatoxin B1 and ochratoxin A. Journal of Public Health and Biological Sciences, 1: 79-85. DOI: 10.13140/ 2.1.2854.3681.

Uyar A, Yener Z and Dogan A (2016). Protective effects of Urtica dioica seed extract in aflatoxicosis: histopathological and biochemical findings. British poultry science, 57(2): 235-245. DOI:10.1080 / 00071668.2015.1129664. PMID: 26947348.

Vayalil PK (2002). Antioxidant and antimutagenic properties of aqueous extract of date fruit (Phoenix dactylifera L. Arecaceae). Journal of Agricultural and Food Chemistry, 50(3): 610-7. PMID: 11804538. DOI: 10.1021/ jf 010716t.

Yildirim E, Yalchinkaya I, Kanbur M, Çnar M and Oruc E (2011). Effects of yeast glucomannan on performance, some biochemical parameters and pathological changes in experimental aflatoxicosis in broiler chickens. Révue de Médicine Vétérinaire, 162 (8): 413-420.

Yunus AW, Razzazi-Fazeli E and Bohm J (2011). Aflatoxin B1 in affecting broiler's performance, immunity, and gastrointestinal tract: A review of history and contemporary issues. Toxins, 3 (6): 566-590. DOI: 10.3390/ toxins3060566.

Zangiabadi H and Torki M (2010). The effect of a Beta-mannanase-based enzyme on growth performance and humoral immune esponse of broiler chickens fed diets containing graded levels of whole dates. Tropical Animal Health Production, 42 (6): 1209 1217. DOI: 10.1007/ s11250-010-9550-1. PMID: 20383608. 